\title{
IMPOTENCIA Y ENFERMEDAD CARDIOVASCULAR: UNA NUEVA PERSPECTIVA EN EL COMPROMISO ASISTENCIAL DEL UROLOGO.
}

\author{
Remigio Vela N a varrete, Juan Vicente G arcia Cardoso, Javier Cabrera Pérez, Carmen \\ Gonzalez Enguita, S. Casado Perez ${ }^{1}$ y J. Farre².
}

Cátedra y Servicio de Urología. Unidad Hipertensiva ${ }^{1}$. Servicio de Cardiología ${ }^{2}$. Fundación Jiménez Díaz. Universidad Autónoma. Madrid. España

\begin{abstract}
Resumen.- O BJETIVO : Revisar las evidencias a portadas hasta el momento que establecen la conexión patogénica entre disfunción eréctil (D.E.) y enfermedad cardiovascular (E.C.V.) y los efectos que este progreso científico ha motivado sobre la consulta urológica tradicional por impotencia.

MÉTO DO S: Las publicaciones más recientes $(2000$ 2004 ) sobre esta patología y el concepto de disfunción endotelial han sido revisadas, mediante los oportunos M edlines, con selección específica de revisiones de conjunto y guías de atención médica.

RESULTADO S: Se confirma que D.E. y E.C.V. comparten factores de riesgo; la unicidad patogénica de ambos procesos, teniendo la disfunción endotelial como problema de fondo; la precocidad de la D.E. en su presentación sobre la E.C.V.; el notable incremento de las investigaciones sobre este tema en los dos últimos años; el cambio de "escenario" en la consulta urológica por impotencia debido a estos hallazgos.
\end{abstract}

\footnotetext{
R. Vela $\mathrm{N}$ a varrete

Fundación Jimenez Díaz

Avda. Reyes Católicos, 2

28040 M adrid. (España)

e-mail: rvela@fjd.es

Trabajo recibido: 7 de octubre 2004
}

CON CLUSIONES: La frecuencia con que D.E. no es una enfermedad de órgano sino el síntoma precoz de disfunción endotelial obliga a cambios en amplitud y profundidad, en la estrategia diagnóstica, pronóstica y de seguimiento de la consulta urológica por impotencia, de extraordinaria importancia individual y sanita ria.

Palabras clave: Disfunción eréctil. Disfunción endotelial. Enfermedad cardiovascular.

Summary.- O BJEC TIVES: To review the evidence currently available to establish the pathogenic connection between erectile dysfunction (ED) and cardiovascular disease (CVD), and the effects this scientific progress has introduced in the classical impotence urology clinic. METHODS: We reviewed the most recent publications about this disease (2000-2004) and the concept of endothelial dysfunction by appropriate M ED LIN E searches, with specific selection of reviews and clinical practice guidelines.

RESULTS: The fact that ED and CVD share risk factors is confirmed; the pathogenic unity of both processes having endothelial dysfunction as the underlying problem; anticipation of ED over CVD in time of presentation; notable increase of research about this issue over the last two years; the change of scenario in the impotence urology clinics due to these findings.

CON CLUSIO N S: The number of cases in which ED is not an organ disease but an early symptom of endothelial dysfunction forces changes in the extent and depth of the diagnostic, prognostic and follow-up strategies in 
the urology impotence clinics of extraordinary importance from both the individual and health-care politics point of view.

Keywords: Erectile dysfunction. Endothelial dysfunction. Cardiovascular disease.

\section{INTRODUCCION:}

La expresión clínica más común de enfermedad cardiovascular en la población actual es la cardiopatía isquémica coronaria que es además la responsable del infarto de miocardio. Se calcula que más de 17 millones de personas mueren al año, en todo el mundo, debido a enfermedad cardiovascular (ECV). La prevalencia de enfermedad coronaria sigue creciendo en los países industrializados y de manera a larmante en los países en desarrollo (1). Cuando se desea conocer si un individuo está en riesgo de enfermedad cardiovascular, y más en concreto de enfermedad coronaria, el clínico actual indaga sobre los conocidos factores de riesgo que incrementan incidencia y prevalencia de este grave proceso, tanto a nivel clínico como de laboratorio y más en concreto sobre el famoso "cuarteto de la muerte": diabetes, obesidad, hipertensión y dislipemia $(2,3)$. Desgraciadamente, a pesar de los esfuerzos realizados hasta el momento para prevenir la enfermedad cardiovascular, la asistencia a las consultas de los $G$ eneralistas de los varones mayores de 40 años es infrecuente, incluso entre los que tienen antecedentes familiares de ECV.

En los últimos años se han acumulado suficientes evidencias para sospechar que la disfunción eréctil puede ser el heraldo, la primera manifestación de disfunción endotelial (4-9). La conexión entre disfunción endotelial, impotencia y ECV estaba firmemente establecida en enfermos con factores de riesgo, como tabaquismo, hipertensión, dislipemia, diabetes, obesidad, síndrome metabólico, etc., pero estos enfermos habitualmente inician su itinerario de consultas médicas en el Generalista u otras especialidades como, Cardiología, Endocrino, etc.. El enorme interés que se ha despertado en los últimos tiempos por consultar con el urólogo, por un lado motivado por el control de la salud prostática, fruto de muy variadas campañas de despistaje del cáncer de próstata, de la HPB, etc., y por otro lado, debido a la precocidad y frecuencia con la que ahora se solici- ta información y ayuda ante los primeros síntomas de disfunción eréctil, han colocado nuestra especialidad en la primera línea de atención a la salud del varón. La calificación del urólogo como "generalista del varón" (10) tiene cada día más sentido y la posición del urólogo en el esquema asistencial de los Sistemas de Salud queda cada día más próxima a la así llamada Medicina Primaria. Si además, consideramos establecida la certeza de que la disfunción eréctil no es en muchas ocasiones una enfermedad de órgano, sino un síntoma precoz de disfunción endotelial, emerge un nuevo compromiso sanitario para el urólogo, que queda comprometido en investigaciones mucho más amplias y en consideraciones médicas de mayor responsabilidad que las que hasta ahora venía desempeñando en la atención del enfermo con impotencia. Puede resumirse la gravedad de este compromiso en el sentido de que el paciente que acude a la consulta del urólogo señalando deficiencias en la erección puede ser un candidato, a corto plazo, de enfermedad coronaria e infarto.

El objetivo de esta revisión es buscar evidencias epidemiológicas y clínicas que establezcan una clara conexión entre disfunción endotelial y disfunción eréctil. Simultáneamente, indagar sobre los factores de riesgo que coinciden en ambos procesos y las recomendaciones que deben establecerse por parte del urólogo para prevenir los riesgos potenciales anunciados por la disfunción eréctil. Finalmente, intentar encontrar una contestación razonada y razonable a las siguientes preguntas: ¿Hasta donde debe llegar el urólogo en sus investigaciones etiopatogénicas de la D.E.?; ¿Hasta donde en sus recomendaciones preventivas y terapéuticas?. ¿Cuándo, cómo y con quien compartir el cuidado del enfermo con impotencia?.

\section{MATERIAL Y METODOS:}

Se han revisado las publicaciones más recientes $(2000-2004)$ relacionadas con este tema cruzando en Medline las siguientes patologías: impotencia, disfunción eréctil, enfermedad cardiovascular, enfermedad coronaria, infarto de miocardio, hipertensión, diabetes y dislipemias. Se han seleccionado fundamentalmente revisiones de conjunto o guías de atención médica, para concluir, después de establecer las evidencias correspondientes, con los aspectos epidemiológicos y con recomendaciones prácticas para el urólogo en ejercicio. 


\section{RESULTADOS:}

\section{A.- Aspectos epidemiológicos de la ECV y}

D.E.: La prevalencia de ECV en la población americana ha sido estudiada recientemente por la American Heart Association (11) confirmándose que aumenta progresivamente con la edad de tal modo que en los varones con 45-54 años la prevalencia es de $6.7 \%$, de $13.1 \%$ a la edad de 55-64 años, del $17.7 \%$ entre 65 74 años y del $18.6 \%$ para los de 75 años o más viejos. Las cifras correspondientes a las mujeres son sustancialmente más bajas pero también progresivamente crecientes con la edad. Una singularidad significativa, en relación con el género, es que a lo largo de su vida el hombre precede a la mujer en incidentes vasculares, siendo la más llamativa la muerte súbita por infarto, que la precede en 20 años. A partir de los 65 la incidencia se dobla en los varones, pero se triplica en las mujeres cuando se compara con la observada en el grupo de edad de 35-65 años. En la mujer, tras la menopausia, la incidencia de ECV aumenta bruscamente siendo tres veces más alta que la misma incidencia en mujeres de la misma edad en estatus premenopáusico. En España, el estudio DO RICA (dislipemia, obesidad y riesgo cardiovascular), llevado a cabo por las Sociedades Españolas para el Estudio de la Obesidad, Arterioesclerosis, Endocrinología y $\mathrm{N}$ utrición, Medicina Interna y Nutrición Comunitaria (12), ha constatado que cerca del $40 \%$ de todas las muertes tienen este origen. Datos más recientes (Banegas et al 2004) (13) confirman que las enfermedades cardiovasculares constituyen la primera causa de muerte en el conjunto de la población española; en el año 2001 causaron 124.245 muertes o lo que es igual el $34,5 \%$ de todas las defunciones acaecidas en España. Esto sitúa a las enfermedades cardiovasculares todavía a gran distancia de la segunda causa de muerte que son los tumores malignos.

Existen numerosos estudios epidemiológicos sobre incidencia y prevalencia de la disfunción eréctil. El Massachusetts Male A ging Study (M M A S) que incluyó a 1.290 varones entre 40 y 70 años mostró una prevalencia global de disfunción eréctil de $52 \%$ etiquetandose de mínima el $17 \%$, moderada el $25 \%$ y completa el 10\% (Felman et al 1995). En España el estudio EDEM (Epidemiología de la Disfunción Eréctil M asculina) realizado con 2.480 varones entre 25 y 70 años de edad mostró una prevalencia global mucho más reducida, del $19 \%$, de la cual el $16 \%$ fue considerada mínima, $2 \%$ moderada y sólo $1 \%$ severa (14).
Si superponemos la información epidemiológica de ambos procesos, como se hace en la Figura 1 comprobamos; una notable superioridad de la prevalencia de la D.E. sobre la ECV; un crecimiento progresivo con la edad. Una lectura añadida de esta imagen es la sugerencia del concepto de "intervalo" caracterizado porque la D.E. precede a la ECV.

B.-Factores de riesgo de ECV y D.E.: La investigación epidemiológica ha podido detectar los factores de riesgo más significativos en la génesis de la ECV. Han sido clasificados en factores de riesgo no modificables como la edad, el sexo, la herencia y modificables (Figura 2). Es importante conocer estos factores de riesgo, tanto clínicos como de laboratorio, porque constituyen el fundamento de las recomendaciones que junto con la medicación pueden ser fundamento y justificación para la terapéutica y prevención primaria de la disfunción endotelial (15-17).

Edad: El varón suele ser más precoz que la mujer en la presentación de ECV. En el estudio del MMAS estos datos a parecen claramente.

Sexo: El varón tiene muchos más riesgos de ECV que la mujer. Incluso después de la menopausia, cuando la incidencia de ECV en la mujer aumenta, este aumento no es tan grande como en los varones hasta que ambos grupos coinciden a la edad de 80 años.

Herencia: La historia familiar es un factor independiente, significativo, de ECV, particularmente en gente joven. La historia paterna de infarto de miocardio antes de los 60 está asociada con un mayor riesgo de ECV.

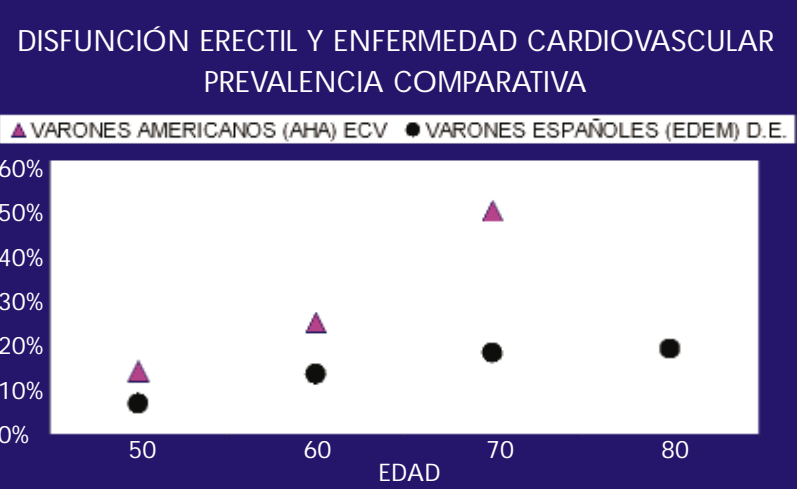

FIG URA 1. Prevalencia comparativa de la D.E., enfermedad cardiovascular en diferentes grupos de población y edad. 
Tabaquismo: La incidencia de ECV se multiplica por seis en las mujeres y por tres en los varones que fuman 20 cigarrillos por día comparado con ciudadanos que nunca han fumado (Prescot et al 1998) (18). Diferentes series muestran que la proporción de varones que fuman es y sigue siendo superior al número de mujeres (19).

Hipertensión: Estudios prospectivos han identificado repetidas veces que el aumento de la tensión arterial tanto de la sistólica como de la diastólica (estudios Framingham) $(20,21)$ incrementa el riesgo de ECV inversamente el control farmacológico de la presión arterial se ha demostrado que reduce el riesgo de ECV tanto en el varon como en la mujer (Chobanian et al 2003) (22).

Dislipemias: Existen sólidas evidencias confirmativas de que las dislipemias incrementan el perfil de riesgo de ECV de manera notable. Las siguientes anormalidades en el perfil lipídico están asociadas con un incremento del riesgo de enfermedad coronaria;. Incremento del colesterol total; bajo colesterol $\mathrm{HDL}$; incremento del indice colesterol total/ HDL; Hipertrigliceridemia; aumento DLBA; aumento del colesterol no HDL, a umento de la A polipoproteina B; y disminución de la A polipoproteina A1 (presente en HDL) (Genes et al 2003) (23). Por otro lado diversos estudios han demostrado que un alto nivel del colesterol HDL tiene efectos protectivos incluso en pacientes con colesterol total elevado; un nivel de colesterol HDL

\begin{tabular}{|c|}
\hline $\begin{array}{c}\text { IMPOTEN CIA Y EN FERMEDAD } \\
\text { CARDIO VASCULAR }\end{array}$ \\
\hline FACTO RES DE RIESG O \\
N O M O DIFICA BLES: \\
Edad \\
Herencia \\
MO DIFICA BLES: \\
Tabaquismo \\
Hipertensión \\
Dislipemia \\
Diabetes \\
O besidad \\
Síndrome metabólico \\
Hipogonadismo \\
\hline
\end{tabular}

FIG URA 2. Factores de riesgo de D.E. y E.C.V.: Clasificación. mayor de $45 \mathrm{mg} / \mathrm{dl}$ se considera como un factor de riesgo negativo. La hipertrigliceridemia es un factor de riesgo independiente, positivamente asociado con sobrepeso, longevidad, diabetes y uso de contraceptivos (Figura 3).

Diabetes: Es un factor mayor, independiente, de riesgo cardiovascular que merece variadas consideraciones que exceden los límites de este trabajo. En el estudio Framingham dobló el riesgo de muerte por enfermedad cardiovascular en los varones y lo triplicó en las mujeres.

O besidad y distribución de la grasa corporal: El estudio Framingham sugiere que el exceso de peso corporal motiva aproximadamente el $3 \%$ de riesgo. La distribución de la grasa también es un importante determinante puesto que los pacientes con grasa abdominal central tienen más riesgo. En España claramente se ha informado sobre la mayor frecuencia de riesgo cardiovascular entre las personas obesas o con sobrepeso (12).

Síndrome metabólico: El síndrome metabólico esta caracterizado por la constelación de obesidad abdominal, hipertensión, diabetes y dislipemia. El síndrome metabólico es calificado también de síndrome de resistencia a la insulina 0 síndrome $x$. Estos pacientes tienen un marcado riesgo de enfermedad coronaria $(24,25)$.

Actividad física: En los últimos años diversos estudios epidemiológicos han demostrado que la acti-

\begin{tabular}{|c|c|}
\hline \multicolumn{2}{|c|}{$\begin{array}{l}\text { IMPO TEN CIA Y EN FERMEDAD } \\
\text { CARDIO VASCULAR }\end{array}$} \\
\hline \multicolumn{2}{|c|}{$\begin{array}{c}\text { M ETA BO LISM O LIPIDICO } \\
\text { (Parámetros a determinar y significado) }\end{array}$} \\
\hline PRUEBA & VA LO RES DE REFEREN CIA \\
\hline $\begin{array}{l}\text { * Colesterol total } \\
\text { * Triglicéridos } \\
\text { * HDL Colesterol } \\
\text { * Cociente } \\
\text { (CO L: Tot./ HDL-CO L) } \\
\text { * Coelsterol de LDL } \\
\text { (Fórmula de Friedenwald) } \\
\text { * Colesterol no HDL }\end{array}$ & $\begin{array}{l}(0-220) \\
(35-200) \\
\text { (Sup.45) N ormalidad (inf.35) Riesgo } \\
\text { (Inf. 4.5) } \\
\text { En función de riesgo individual. } \\
\text { En función del nivel de riesgo individual }\end{array}$ \\
\hline \multicolumn{2}{|c|}{$\begin{array}{l}\text { El mejor escenario consiste en: BAJO CO LESTERO L TO TA L: } \\
\text { BAJO CO LESTERO L DL (colesterol malo!): A LTO CO LESTERO L HDL. } \\
\text { O tros estudios analíticos indicados si se sospechan factores de } \\
\text { riesgo por diabetes, hipogonadismo, insuficiencia hepática, etc. }\end{array}$} \\
\hline
\end{tabular}

FIG URA 3. M etabolismo lípido: Parámetros a incluir y significa do clínico. 
vidad física baja el riesgo de ECV cuando comparados con individuos sedentarios (26).

Estatus hormonal: En la mujer, la terapéutica sustitutiva de deficiencias estrogénicas ha sido motivo de controversia en los últimos años. En el varón, algunos estudios han demostrado que altos niveles de testosterona tienen un efecto positivo sobre la salud cardiovascular (Engilsh et al 2000, Zemuda et al 1997). $(27,28)$

Otros factores de riesgo: A buso del alcohol, mientras que el uso moderado de vino reduce el riesgo de ECV en ambos sexos (French Paradox). La mayoría de los beneficios del vino parecen estar mediados por un incremento del colesterol HDL, aunque se han implicado otros efectos antioxidantes, antitrombótico y antiinflamatorios.

Los factores de riesgo advertidos en la disfunción eréctil coinciden ampliamente con los factores de riesgo señalados en la ECV. En el estudio MMAS, el $34.8 \%$ de los varones entre 40-70 años tenían disfunción eréctil moderada a completa con un $15 \%$ en los de 70 años. El riesgo de D.E. aumentó notablemente con la edad de tal modo que se multiplicó por 3.6 en los varones con 50-59 años frente a los de 18-29 a ños. Este mismo estudio mostró mayor prevalencia de la D.E. en pacientes con enfermedad cardiovascular y se ha podido confirmar que existe correlación positiva entre la gravedad de la D.E. y el número de vasos afectos en la enfermedad coronaria. Igualmente, la prevalencia de la D.E. en paciente con hipertensión es 1.5 veces más alta que en la población general, en cualquier grupo de edad. Por otro lado, la prevalencia de la D.E. es más elevada en fumadores, diabéticos y pacientes con hipercolesterolemia. En definitiva, la investigación epidemiológica de ambos procesos, aparentemente tan distintos, confirma que deben tener un fundamento común. Una vez más, los argumentos a favor de esta unicidad patológica, versus dos enfermedades distintas, serían los siguientes: Comparten factores de riesgo comunes; a mayor severidad de la enfermedad vascular mayor severidad de la disfunción eréctil; y la D.E. es más común en pacientes con enfermedad vascular. Verosimilmente D.E. y ECV comparten un fundamento patogénico común; es a este potencial nexo de unión de ambos procesos a lo que calificamos de enfermedad endotelial.

\section{C.- Impotencia como heraldo de enferme-} dad cardiovascular: No existe información precisa sobre este tema. Es evidente que el urólogo no ha indagado hasta el momento, de manera regular, sobre los riesgos inherentes a ECV en el enfermo que consulta por D.E.. N o obstante se tiene información de las patologías sistémicas no conocidas previamente que han sido descubiertas por urólogos en estos pacientes; por ejemplo, una alta tasa de glucemia o colesterol. En general esta perspectiva no ha sido exhaustivamente investigada desde la consulta urológica y faltan estudios longitudinales que definan con precisión la continuidad del proceso y la dimensión del "intervalo".

La relación entre ECV y D.E. ha sido demostrada por un lado confirmando la incidencia de D.E. en cardiopatas y al contrario. Un reciente análisis de Kloner (29) ha mostrado que el $75 \%$ de los varones con ECV estable tienen disfunción eréctil. Por el contrario, los pacientes que consultan por D.E. pueden tener más factores de riesgo de ECV que la población normal. Consideración especial merece un reciente trabajo (El-Sakka et al 2004) (30) en el que se investigó la incidencia de ECV específicamente desde la consulta urológica en 417 pacientes que con una edad media de $59.1 \pm 10.3$ años consultaron por E.D.. Encontraron que, globalmente, el $92.1 \%$ de todos los pacientes con D.E. tenían uno o más factores de riesgo de enfermedad coronaria. Por otro lado a mayor D.E. mayor edad y mayor incidencia de riesgos de ECV; diabetes, hipertensión, dislipemias y desórdenes psicológicos estuvieron presentes en el 75\%, 40\%, 45\% y $8 \%$ de los pacientes respectivamente.

La prevalencia de D.E. en pacientes con ECV puede considerarse en una media del $60 \%$ y el amplio rango observado (42-70\%) puede explicarse por muchos motivos generales y específicos; diferencia en las características de la población estudiada; definición de D.E.; pruebas utilizadas para el diagnóstico de la ECV; cronicidad del proceso ECV ya que la D.E. es de menor incidencia en pacientes con procesos agudos que en aquellos con enfermedad crónica. (Montorsi et al 2004). A su vez los mismos argumentos son válidos para reconocer la confusa prevalencia de ECV en pacientes con D.E. porque el resultado final depende de la técnica empleada para esta investigación. La mayoría de las exploraciones están basadas en la prueba de esfuerzo y en este sentido, cuando el paciente con D.E. carece de antecedentes de enfermedad vascular habitualmente es positiva entre un $5.1 / 19.7 \%$ de los casos con una media del $12 \%$. Por el contrario si el paciente tiene antecedentes positivos de ECV el porcentaje de pruebas positivas es mucho 
mayor (20-56\%) (Kim et al 2001). Puede recurrirse a la angiografía coronaria y en este sentido el grupo de Montorsi (2003) (9) estudió 300 pacientes con enfermedad cardíaca documentada entre ellos un $49 \%$ con D.E.y se advirtió claramente la prioridad de la D.E., incluso con tres años de antelación, sobre la isquemia cardiaca. Finalmente si se investiga la enfermedad vascular periférica mediante angiografía se ha reconocido hasta un $75 \%$ de aumento de riesgo de D.E. en aquellos que la sufren (Brumentals et al 2003) (33). Puede concluirse desde un punto de vista práctico que la D.E. es la manifestación clínica de una enfermedad cardiovascular que afecta a la circulación peneana de igual modo que el angor e infarto de miocardio es la manifestación clínica de un padecimiento vascular que afecta a la circulación coronaria. Esta afirmación justificaría, desde el punto de vista práctico, que los pacientes con E.D. sean considerados como potenciales enfermos vasculares con todas sus consecuencias.

\section{D.- Enfermedad endotelial como nexo pato- génico comun de D.E. y ECV.}

Kloner (29) se pregunta; ¿Q ué es la disfunción endotelial como fundamento patogénico común de la ECV y D.E.?. Se trata de un comportamiento anormal del endotelio, con una respuesta inapropia$d a$, fundamentalmente en su mecanismo de vasodilatación, frente a diferentes estímulos. Desde el punto de vista funcional lo que caracteriza a la disfunción endotelial es la incapacidad del endotelio de producir N 0 (óxido nítrico) y conseguir la vasodilatación de manera satisfactoria $(34,35)$. N umerosos estudios bioquimicos, histoquímicas y fisiológicos indican que el $\mathrm{NO}$ es un mediador esencial en la función eréctil. El NO es sintetizado como producto catalítico de conversión de L-arginina a L-citrulina por la enzima No-sintasa (N OS). Se han descrito tres isoenzimas de la NOS que difieren en su estructura molecular y en sus propiedades biológicas; la neuronal (NOSn); la endotelial (N O Se); y la inducible (N O Si), tipicamente asociadas a macrófagos y otras células que participan en la función inmune (Figura 4). Se conoce bastante sobre el NO producido por la NOSn pero la participación del $\mathrm{NO}$ endotelial se va desvelando progresivamente por investigaciones en ratones transgénicos carentes de NOSn y por tinciones inmunohistoquímicas de la $\mathrm{N} O \mathrm{Se}$ en el endotelio de los sinosoides cavernosos, en el músculo liso trabecular y en las paredes de las arterias elicinas. Se sospecha que a pesar de la importancia del NOSn es necesaria una función endotelial intacta para conseguir una función eréctil correcta y que es precisamente esta disfunción endotelial la que sirve de fundamento patogénico común a muchos padecimientos vasculares.

Hay varias formas de reconocer esta deficiencia en clínica cardiológica (test de Treasure, prueba de la vasodilatación compensatoria de la isquemia braquial) pero en clínica urológica no tenemos ningún procedimiento específico para estos fines. Justifica está ausencia de marcadores clínicos el interés por un marcador molecular, analítico. Una primera aportación ha sido realizada por nuestro grupo (García Cardoso. J.V) (36) y hay otras iniciativas en marcha. Es preceptivo, en cualquier caso, incluir en el examen analítico todos aquellos marcadores de riesgo endotelial sospechados, además del perfil lipídico, como glucemia y hemoglobina glucoxilada en potenciales diabéticos, perfil hormonal (Testosterona-LH-Prolactina-DHEA-S) en hipogonadismo, prueba de función hepática y otros estudios (Figura 3).

\section{RECOMENDACIONES:}

Confirmada que la disfunción eréctil puede ser el heraldo de disfunción endotelial, el urólogo que atiende por primera vez al paciente consultando por disfunción eréctil, a cualquier edad, sin antecedentes claros de ECV, adquiere una responsabilidad que da a su práctica médica una nueva perspectiva hasta hace años ignorada. Este compromiso acerca al uró-

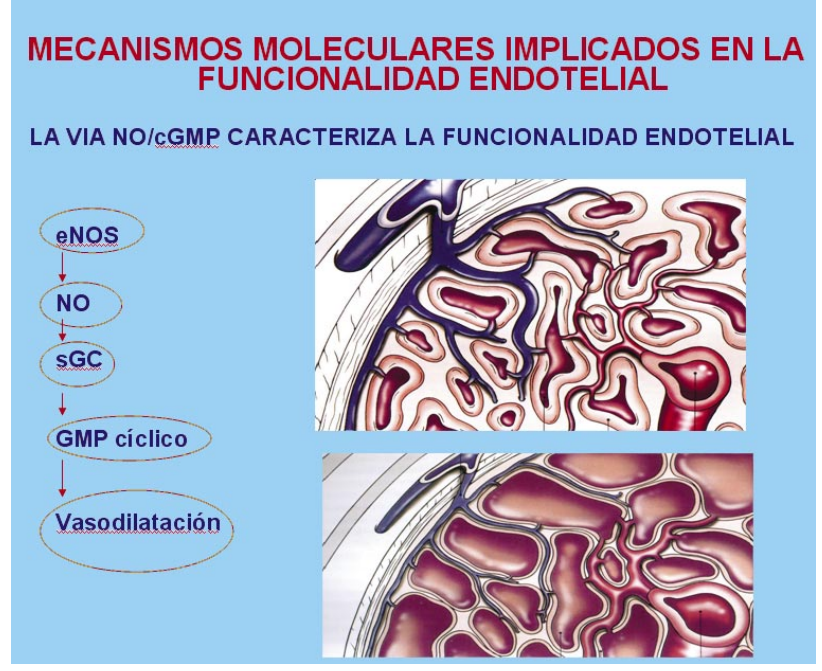

FIG URA 4. Función endotelial. Esquema factores involucrados en la vasodilatación. 
logo, de manera llamativa, a nuevos protagonismos sanitarios que progresivamente han sido mejor definidos en los últimos años calificando su actividad con el título del "Generalista del Varón". Efectivamente, la consulta del enfermo con disfunción eréctil, sin otras patologías coincidentes, ha motivado un extraordinario cambio de estrategia asistencial ya que obliga al urólogo a estudiar al paciente de una manera más integradora y cabal que lo que hasta ahora venía siendo un examen fundamentalmente dirigido al órgano enfermo. La disfunción eréctil no es una enfermedad de órgano, es una enfermedad sistémica, multifactorial, en la que al menos dos patologías coincidentes deben ser indagadas; disfunción endotelial, y deficiencias androgénicas propias del varón que envejece $(27,28)$. Seguramente en un futuro próximo habrá otros aspectos que obliguen a un estudio incluso más amplio que el que aquí se propone presidido por el factor de riesgo más común en estos padecimientos; el envejecimiento (37). Las siguientes recomendaciones intentan contestar a las cuestiones inicialmente planteadas: ¿Hasta donde debe llegar el urólogo en sus investigaciones etiopatogénicas, en sus recomendaciones preventivas y terapéuticas, y cómo, cuando y con quién debe compartir el cuidado del enfermo con impotencia?.

\section{O bligatoriedad y limites de la actuación urológica.}

Moyad (3) considera que hay numerosas

\begin{tabular}{|c|} 
IMPOTENCIA Y EN FERMEDAD \\
CARDIO VASCULAR \\
\hline RAZO N ES PARA UN A VA LO RACIÓ N IN TEG RAL \\
EN LA CON SULTA URO LÓ G ICA \\
(M oyad, M.A. 2004) \\
* La ECV es la primera causa de muerte en todos los países \\
industrializados \\
* La alta incidencia de ECV en la población añosa que \\
consulta por problemas urológicos. \\
* La ECV es también la causa más común de muerte en \\
los grandes ensayos clínicos de prevención del cáncer \\
de próstata. \\
* La coincidencia de factores de riesgo y mecanismos \\
patogénicos entre ECV y enfermedades urológicas, muy \\
especielmente con la D.E.
\end{tabular}

FIG URA 5. D.E. Razones para una valoración integral en la consulta urológica. razones para que el urólogo haga un acercamiento integral a los pacientes con disfunción eréctil. Son las siguientes: La ECV es la causa primaria de muerte de los varones en todas partes del mundo y muy especialmente en los países industrializados, y el urólogo no puede quedar al margen de este compromiso diagnóstico y preventivo: La ECV es también causa primera de muerte entre los varones añosos que consultan por problemas urológicos. Tanto es así que la ECV es la causa más común de muerte incluso en los grandes estudios de prevención del cáncer de prósta ta realizados hasta el momento: Coexisten factores de riesgo y mecanismos patogénicos comunes entre la ECV y algunas condiciones 0 enfermedades urológicas y muy en especial con el CAP y la HPB. (Figura 5)

Puesto que la salud cardiovascular se refleja en la salud urológica es imperativo para el urólogo discutir la probabilidad de comorbilidades y mortalidades con estos pacientes, actitud que no va a reducir la importancia de otras enfermedades urológicas, pero va a colocar los riesgos del paciente en la perspectiva apropiada. Y los pacientes necesitan estas guías, más que nunca, porque la expectación de vida continua aumentando y la coincidencia potencial de comorbilidades es condición inexcusable del varón que envejece.

\begin{tabular}{|l|r|r|}
\hline Edad & 57 & $\mathrm{Años}$ \\
\hline Colesterol $\mathrm{DL}$ & 166 & $\mathrm{mg} / \mathrm{dl}$ \\
\hline Colesterol HDL & 65 & $\mathrm{mg} / \mathrm{dl}$ \\
\hline Triglicéridos & 109 & $\mathrm{mg} / \mathrm{dl}$ \\
\hline Presión arterial sistólica & 147 & $\mathrm{mmHg}$ \\
\hline Consumo de cigarrillos & $\mathrm{NO}$ & \\
\hline Diabetes mellitus & $\mathrm{NO}$ & \\
\hline Infarto de miocardio en historia familiar & $\mathrm{SI}$ & \\
\hline
\end{tabular}

Su riesgo de padecer un infarto de miocardio es de $\mathbf{4 . 6 3 \%}$ en los próximos diez años.

Esto le incluye en el 30 quintil (quinto) de la población de varones de mediana edad (véase la figura)

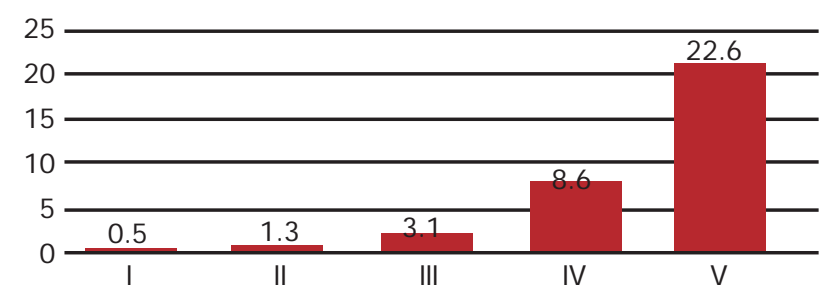

FIG URA 6. Cálculo del riesgo cardiovascular (PRO CAM). 
Pero esta perspectiva tiene ta mbién sus límites y el urólogo tiene que descubrir, advertir y orientar hacia otras especialidades con mayor experiencia y compromiso en este tipo de patología. Es evidente que al urólogo no le corresponde: realizar una evaluación cardiovascular en una persona que consulta por un padecimiento no vascular; extremar indagaciones sobre potenciales patologías diabéticas o del metabolismo lipídico; hacer exámenes especiales relacionados con una hipertensión primaria; etc. Por el contrario el urólogo está obligado a; realizar una historia clínica de mayor amplitud investigando con rigor comorbilidades coincidentes, medicaciones tomadas por el enfermo, solicitar la bioquímica clínica precisa para descartar factores de riesgo mayores, al menos relacionados con hiperglucemia, trastornos lípidicos, incluyendo valoraciones orientativas del riesgo cardiovascular, con las advertencias pertinentes. En aquellos pacientes en los que existe conocida ECV está especialmente obligado a utilizar con el mayor conocimiento y cautela la medicación oral con inhibidores de la PDE5 y a valorar con extremado rigor el riesgo inherente a cualquier actividad sexual, con 0 sin medicación, a ser posible en atención compartida con el cardiólogo.

Evaluación del riesgo cardiovascular: Hay varios modelos para calcular el riesgo de ECV utilizando diversos parámetros. El más conocido es el Framingham que incluye las siguientes referencias: la edad, el nivel de colesterol total, el tabaquismo; la presión arterial sistólica y el nivel de HDL (20). Para calcular el baremo se comienza por la edad del paciente, comprobando en la tabla de referencias los puntos que le corresponden, a los que se van añadiendo, en pasos sucesivos, el colesterol total en función de la edad, la adicción al tabaco en función de la edad, el nivel de HDL (Figura 6). Hay otros baremos como el Procam y el Score (Systemic Coronary Risk Evaluation) para estos fines.

Esta sencilla valoración del riesgo cardiovascular, que puede asumir el urólogo, no modifica en gran modo sus estrategias diagnósticas generales. De acuerdo con las recomendaciones contenidas en el texto de la OMS el paciente con conocida ECV, o sospechada, debe ser especificamente valorado en este sentido por su cardiólogo (38).

¿Cómo se deben utilizar los PDE5 en los enfermos cardiopatas?. El tema se refiere fundamentalmente a los enfermos con isquemia cardíaca. No parece que haya contraindicaciones fundamentales y se ha demostrado que no alteran la respuesta al cateterismo 0 al test de esfuerzo e incluso la mejoran. Efectivamente estos productos motivan vasodilatación coronaria y periférica simultáneamente por lo que es de esperar que interactuen con otras medicaciones que afectan la tensión arterial. En general son seguros cuando se usan en combinación con la mayor parte de la medicación vasodilatadora, incluyendo los bloqueantes del calcio, los inhibidores de la enzima de conversión y los bloqueantes de los receptores de angiotensina. Sin embargo, están contraindicados en pacientes tomando cualquier tipo de nitratos debido al efecto potenciador sobre la bajada de tensión. Esta respuesta excesiva por la coincidencia terapéutica es verosímilmente el resultado de la interacción sinérgica de ambos fármacos en la vía del óxido nítrico-guanilil cyclasa-CGMP. Los nitratos orgánicos sirven como donantes de óxido nítrico que estimulan la guanilil cyclasa y aumentan la concentración intracelular de GMPC, mientras que los PDE5 impiden la destrucción de CGMP (39).

En los individuos que toman nitritos y tienen una crisis aguda de angor durante la actividad sexual no debe utilizarse nitroglicerina y deben ser tratados con otras medicinas antiisquémicas, como betabloqueantes, bloqueantes de calcio, morfina y estatinas (29).

La interacción hemodinámica potencial entre IOS PDE5 y alfa adrenérgicos es de particular interes para los urólogos. En los varones con HPB los alfabloqueantes como Doxazosina, Tamsulosina, Terazosina y A lfuzosina son frecuentemente utilizados para aliviar los síntomas urinarios. Como este grupo de población tiene a su vez una elevada incidencia de disfunción eréctil pueden coincidir ambos tratamientos. La combinación de Sildenafil y alfabloqueantes puede motivar hipotensión postural. Dosis de Sildenafil superior a 25 mg aconsejan que no se tome un alfabloqueante al menos en las 4 horas siguientes. El uso de Vardenafil esta contrandicado en pacientes tomando alfabloqueantes debido a la producción de hipotensión ortostatica observada en pacientes tomando Vardenafil y Terazosina. Tadalafil en combinación con Doxazosina y Tamsulosina ha sido investigado por Kloner (39) confirmando que $20 \mathrm{mg}$., de Tadalafil bajan la presión arterial combinados con $8 \mathrm{mg}$., de Doxazosina pero esta interacción no es hemodinamicamente relevante cuando coincidiendo con $0.4 \mathrm{mg}$ de Tamsulosina por lo que este fármaco puede ser considerado como el alfabloqueante preferido para el tra- 
FIG URA 7. Clasificación de Pricenton sobre el grado de riesgo.

\begin{tabular}{|c|c|c|}
\hline \multicolumn{3}{|c|}{$\begin{array}{l}\text { ACTIVIDAD SEXUAL Y EN FERMEDAD } \\
\text { CARDIO VASCULAR } \\
\text { DACIO N ES DE PRICEN TO N (DeBusk et al } 2000)^{41}\end{array}$} \\
\hline $\begin{array}{l}\text { GRADO } \\
\text { DE RIESGO }\end{array}$ & CATEG O RÍA DE E.C.V. & RECO MEN DACIO N ES \\
\hline BAJO & $\begin{array}{l}\text { A síntomáticos con <3 factores de riesgo de Enf. } \\
\text { Coronaria, Hipertensión controlada } \\
\text { Angor estable, moderado, Revascularización satis- } \\
\text { factoria, Infarto no complicado pasadas } \\
6-8 \text { semanas. Moderada Enf. Valvular. Fracaso car- } \\
\text { díaco congestivo DVI=N YHA *Clase I }\end{array}$ & $\begin{array}{l}\text { Generalistas } \\
\text { Terapias de primera línea } \\
\text { Evaluaciones intervalos regulares } 6-12 \text { meses }\end{array}$ \\
\hline IN TERM EDIO & $\begin{array}{l}\geq 3 \text { factores de riesgo mayores. Angor estable, } \\
\text { moderado. Reciente infarto (entre 2-6 semanas) } \\
\text { FCC/ DVI=N YHA Clase II. A usencia de secuelas } \\
\text { extracardiacas (ACVA) }\end{array}$ & $\begin{array}{l}\text { Precisan estudios cardiovasculares (test de esfuerzo, } \\
\text { ecocardiograma) Revaloración de categoría (alto o } \\
\text { bajo riesgo) según resultados del estudio cardiovas- } \\
\text { cular }\end{array}$ \\
\hline A LTO & $\begin{array}{l}\text { Angor inestable o refractario Hipertensión no con- } \\
\text { trolada FCC / DVI=HYHA Clase Ill. } \\
\text { Infarto o ACVA muy reciente (<2 semanas). } \\
\text { A rritmias de alto riesgo. } 0 \text { tras condiciones de alto } \\
\text { riesgo. }\end{array}$ & $\begin{array}{l}\text { Prioridad manejo por cardiólogo } \\
\text { Tratamiento diferido según evolución }\end{array}$ \\
\hline
\end{tabular}

tamiento de la sintomatología prostática en pacientes que reciben Tadalafil.

¿Cómo se trata el paciente con ECV que consulta por D.E.?. ¿Debe el urólogo ocuparse de estos pacientes 0 ceder la responsabilidad al cardiólogo 0 al generalista?.
El manejo de la D.E. y de la disfunción sexual en el paciente cardiopata ha sido descrito con precisión en recientes guías de comportamiento (Debust et al 2000; Cheiclin et al 1999) (39-41), siendo la más aplicada en Estados Unidos la conocida como la guía de consenso de Priceton (42). De acuerdo con estas

\section{ACTIVIDAD SEXUAL COMO FACTOR DE RIESG O*}

$(\text { Y. Drory 2002) })^{42}$

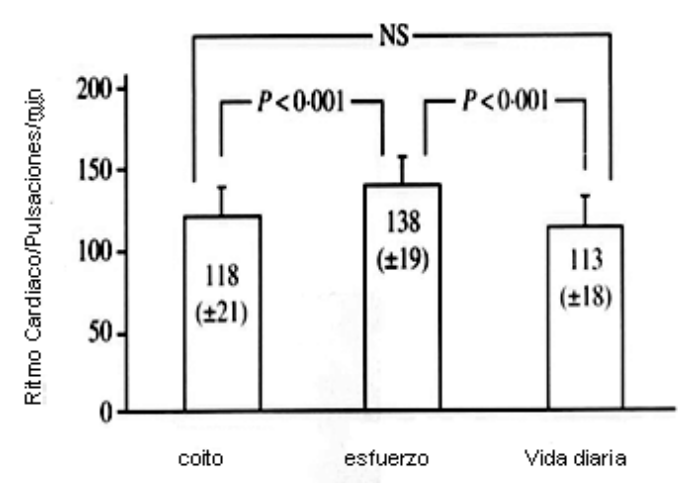

Pulsaciones por minuto durante la actividad sexual, ejercicio y actividad normal diaria.

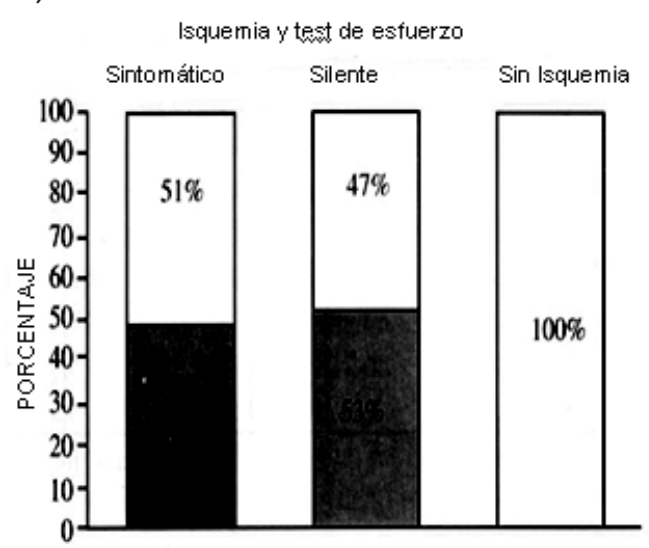

Isquemia cardiaca motivada por la actividad sexual en pacientes con test de esfuerzo negativo y positivo 
guías de evaluación, los pacientes son clasificados en una de estas tres categorías; bajo riesgo, indeterminado 0 intermedio y alto riesgo. En el grupo de bajo riesgo se incluyen aquellos pacientes asintomáticos que tienen menos de 3 factores de riesgo de ECV, hipertensión controlada, angor medio estable, revascularización coronaria con éxito, antecedentes de infarto de miocardio no complicado de más de seis u ocho semanas de evolución, enfermedad valvular moderada. Solo estos enfermos son los que deberían ser tratados por el urólogo o su médico general, con cualquiera de las terapias de primera línea (Figura 7). Sin embargo los pacientes pertenecientes al grupo 20 3 incluyen riesgos suficientes como para desaconsejar la actividad sexual hasta que sean valorados por el Cardiólogo ya que la actividad sexual es en si misma un factor de riesgo (aumenta el ritmo cardíaco, promueve arritmias, motiva angor e infartos (Figura 8) con o sin farmacología PDE-5 coincidente).

\section{CONCLUSIONES:}

D.E. y E.C.V. comparten frecuentemente factores de riesgo comunes, y se comportan como la misma enfermedad con expresión clínica distinta en función de los territorios vasculares afectos, con distinta cronología, sucesiva o coincidente, pero generalmente precedida por el síntoma disfuncional eréctil con un "intervalo" variable.

El fundamento patogénico común de éstos procesos es la disfunción endotelial, proceso sistémico, generalizado, de expresión clínica variable, dominando en algunos casos las lesiones anatómicas groseras identificables mediante procedimientos de imagen, y en otros, la alteración funcional más elusiva en su reconocimiento clínico.

La disfunción endotelial tiene como fundamento bioquímico limitaciones en la funcionalidad del $\mathrm{N} O$ producido por el endotelio, incapaz de conseguir la respuesta vasodilatadora del músculo liso endotelial en circunstancias fisiológicas o patológicas.

Frente a este escenario, de mayor riesgo que el tradicionalmente atribuido a la D.E., el urólogo, principal proveedor de asistencia médica en este tipo de patología, debe actuar con mayor rigor en sus investigaciones etiopatogénicas, incluyendo las interconsultas oportunas, mayor cautela en sus recomendaciones terapéuticas y con mayor amplitud informativa y perspectiva de salud general que ante una simple enfermedad de órgano.

\section{BIBUOGRAFIA y LECTURAS RECOMENDADAS (*lectura de interés y **lectura fundamental)}

*1. KIRBY, R.S.; KIRBY, M.G.; FARAH, R.N.: "Men's Health". Isis Medical Media/Oxford, 1999.

2. FODOR, J.G.; TZEROVSKA, R.: "Coronary heart disease: is gender important?".JMHG. Vol. 1, 1: 32. 2004.

*3. MOYAD, M.A.: "Introduction to risk assessment and serum risk markers for the prevention of coronary heart disease and other potential conditions that impact men's health, part II: What do I tell my patients?". Urol. Clin. N. Am., 31: 199. 2004.

4. VIRAG, R.; BOUILLY, P.; FRYDMAN, D.: "Is impotence an arterial disorder? A study of arterial risk factors in 440 impotent men". Lancet, 1: 181. 1985.

5. FELDMAN, H.A.; GOLDSTEIN, I.; HATZICHRISTOU, D.G. y cols.: "Impotence and its medical and psychosocial correlates: results of the Massachusetts Male Aging Study." J. Urol., 151: 54. 1994.

6. GREENSTEIN, A.; CHEN, J.; MILLER, H. y cols.: "Does severity of ischemic coronary disease correlate with erectile function?”. Int. J. Impot. Res., 9: 123. 1997.

*7. KIRBY, M.; JACKSON, G.; BETTERIDGE, J. y cols.: "Is erectile dysfunction a marker for cardiovascular disease?". Int. J. Clin. Pract., 55: 614. 2001.

8. BURCHARDT, M.; BURCHARDT, T.; BAER, L. y cols.: "Hypertension is associated with severe erectile dysfunction”. J. Urol., 164: 1188. 2000.

**9. MONTORSI, F.; BRIGANTI, A.; SAJONIA, A. Y cols.: "Erectile dysfunction prevalence, time of onset and association with risk factors in 300 consecutive patients with acute chest pain and angiographically documented coronary artery disease". Eur. Urol., 44: 360. 2003.

10. VELA NAVARRETE, R.; ANDERSEN, J.T.; BOROWKA, A. y cols.: "El Futuro de la Urología en Europa: Una perspectiva desde la Asociación Española de Urología". Actas Urol. Esp., 3: 156. 2001.

11. American Heart Ass. Heart disease and stroke statistic: update: www.american heart.org/presenter/downloaddoble/heart. 2004.

12. ARANCETA, J.; FOC, M.; GIL, B. y cols.: "Obesidad y riesgo cardiovascular. Estudio Dorica". Ed. Medica Panamericana. Laboratorios Lacer. 2004.

13. BANEGAS, J.R.; VILLAR, F.; RODRÍGUEZ ARTALEJO, F.: "Evaluación del riesgo cardiovascular en la población española". Risk factors, 13: 155. 2004.

*14. MARTIN-MORALES, A.; SANCHEZ-CRUZ, J.J.; SAENZ DE TEJADA, I.: "Prevalence and independent risk factors for erectile dysfunction in Spain. Results of the EDEM study (epidemiología de la DE masculina)". J. Urol., 166: 569. 2001.

15. LUE, T.F.: "Erectile dysfunction". New Engl. J. Med., 342: 1802. 2000.

*16. FELDMAN, H. A.; JOHANNES, C. B.; DERBY, C. A. y cols.: "Erectile dysfunction and coronary risk factors: prospective results from the Massachusetts Male Aging Study". Prev. Med., 30: 328. 2000.

17. DE ANGELIS, L.; MARFELLA, M.A.; SINISCALCHI, M. y cols.: "Erectile and endothelial dysfunction in type II diabetes: a possible link". Diabetología, 44: 1155. 2001.

18. PRESCOTT, E.; HIPPE, M.; SCHNOHR, P. y cols.: "Smoking and the risk of myocardial infarction in women and men: Longitudinal population study". Br. Med, J., 316: 1043. 1998.

19. SHABSIGH, R.; FISHMAN, I.J.; SCHUM, C. y cols.: "Cigarette smoking and other vascular risk factors in vasculogenic impotence". Urology, 38: 227. 1991.

20. ANDERSON, K.M.; WILSON, P.W.; ODELL, P.M. y cols.: "An updated coronary risk profile. A statement for health professionals". Circulation, 83: 356. 1991. 
**21. MOYAD, M.: "Introduction to risk assesment and serum risk markers for prevention of coronary heart disease (Part I \& II)". Urol. Clin. N. Am., 31: 199. 2004.

22. CHOVANIAN, A.V.; BAKRIS, G.L.; BLACK, H.R. y cols.: "The seventh report of the Joint National Committee on Prevention, Detection, Evaluation, and Treatment of High Blood Pressure". J. Am. Med. Assoc., 289: 2560. 2003.

23. GENEST, J.; FRIHLICH, J.; FODOR, G. y cols.: "The working group on Hypercholesterolemia and Other Dyslipidemias. Recommendations for the management of dyslipidemia and the prevention of cardiovascular disease". CMAJ., 169: 921. 2003.

24. KAPLAN, N.M.: "Obesity, insulin and hypertension". Cardiovasc Risk Factors, 4: 133. 1994.

25. LAKKA, H.M.; LAAKSONEN, D.E.; LAKKA, T.A. y cols.: "The metabolic syndrome and total and cardiovascular disease in middle-aged men". J. Am. Med. Assoc., 288: 2709. 2002.

26. POWELL, K.E.; THOMPSON, P.D.; CASPERSEN, C. y cols.: "Physical activity and the incidence of coronary heart disease". Annu. Rev. Public Health, 8: 253. 1987.

27. ENGILSH, K.M.; MANDOUR, O.; STEEDS, R.P. y cols.: "Men with coronary artery disease have lower levels of androgens than men with normal coronary angiograms". Eur. Heart J., 21: 890. 2000.

28. ZMUDA, J.M.; CAULEY, J.A.; KRISKA, A. y cols.: "Longitudinal relation between endogenous testosterone and cardiovascular disease risk factors in middle aged men: a 13year follow-up of former". Multiple Factor Intervention Trial participants. Am. J. Epidemiol. 146: 609. 1997.

*29. KLONER, R.A.; MULLIN, S.; SHOOKT y cols.: "Erectile dysfunction in the cardiac patient: How common and should we treat?". J. Urol., 170: 46. 2003.

30. EL-SAKKA, A.; MORSY, A.M.; FAGIH, B.I. y cols.: "Coronary artery risk factors in patients with erectile dysfunction". J. Urol., 172: 251. 2004.

**31. MONTORSI, P.; ROUMEGUERE, T.; MONTORSI, F. y cols.: "Is there a link between Erectile Dysfunction and Coronary Artery Disease?”. EAU Update Series, 2: 43. 2004.

32. KIM, S.W.; PAICK, J.S.; PARK, D.W. y cols.: "Potential predictors of asymptomatic ischemic heart disease in patients with vasculogenic erectile dysfunction" Urology, 58: 441. 2001.

33. BRUMENTALS, W.A.; GOMEZ-CAMINERO, A.; VANNAOOAGARI, V.: "Is erectile dysfunction predictive of peripheral vascular disease?". Aging Male, 6: 217. 2003.

34. KIM, N.; AZADZOI, K.M.; GOLSTEIN, I. y cols.: "Nitric oxide-like factor mediates nonadrenergic-noncholinergic neurogenic relaxation of penile corpus cavernosum smooth muscle". J. Clinc. Invest., 88: 112. 1991.

35. LUE, T.: "Erectile dysfunction". N. Engl. J. Med., 342: 1802. 2000.

36. GARCIA CARDOSO, J.V.: "Determinacion del sistema del oxido nitrico/GMP/ ciclico en células mononucleares obtenidas de pacientes con disfunción eréctil". Tesis Doctoral. UAM. 2004.

37. VELA NAVARRETE, R.: "Envejecimiento y Urologia". Actas Urol. Esp., 2: 91. 2000,

*38. "Erectile Dysfunction". World Health Organization. Oxford U.K. Health Publication LTD. Plymouth, 2000.

39. KLONER, R.A.; EMMICK, J.; BEDDING, A. y cols.: "Pharmacodynamic interactions between tadalafil and nitrates". J. Am. Coll. Cardiol., 39: 291A. 2002.

40. CHEITLIN, M.D.; HUTTER, A.M.Jr.; BRINDIS, R.G. y cols.: "ACC/AHA expert consensus document. Use of Sildenafil (Viagra) in patients with cardiovascular disease". J. Am. Col. Cardiol., 33: 273. 1999.

**41. DEBUSK, R.; DRORY, Y.; GOLDSTEIN, I. y cols.: "Management of sexual dysfunction in patients with cardiovascular disease: recommendations of the Princeton Consensus Panel". Am. J. Cardiol., 86: 175. 2000.

42. DRORY, Y.: "Sexual activity and cardiovascular risk". Eur. Heart J. Supplements, H13-H18. 2002. 